\title{
AVALIAÇÃO DA PREVALÊNCIA DE ENTEROPARASITOSES E A EFICÁCIA DO CONSUMO DE Lactobacillus spp. PARA O CONTROLE DE INFECÇÕES \\ PARASITÁRIAS.
}

\section{ASSESSMENT OF THE PREVALENCE OF ENTEROPARASITOSES AND THE EFFECTIVENESS OF THE CONSUMPTION OF Lactobacillus spp. FOR THE CONTROL OF PARASITIC INFECTIONS.}

\author{
Franklin Emmanuel B. Pereira Filho1*
}

1 - Biomédico responsável pelo setor de virologia no Laboratório Municipal de Referência Regional de Serrinha (LACEN/BA-LMRR Serrinha) e professor do curso de Biomedicina na Faculdade Pitágoras de Serrinha

\section{Resumo:}

Parasitoses intestinais são doenças transmitidas aos seres humanos, que contribuem para diversas alterações metabólicas no corpo do hospedeiro, estas infecções são associadas a hábitos que potencializam o processo de disseminação das doenças e ocorrem principalmente em regiões menos desenvolvidas, contudo sugere-se que probióticos, em especial o Lactobacillus spp. pode aumentar a atividade da microbiota intestinal, atuando contra infecções parasitárias. $O$ presente estudo trata-se de uma revisão bibliográfica, qualitativa, onde foram utilizadas 39 referências com o objetivo de avaliar a prevalência de enteroparasitoses e fatores que determinam essa variação, bem como a eficácia do Lactobacillus spp. no controle de infecções parasitárias. Concluiu-se que a prevalência de enteroparasitoses está relacionada a fatores socioeconômicos e sanitários, e que embora pouco se saiba ainda sobre o mecanismo de ação, os lactobacillus spp. mostraram-se eficazes nos experimentos realizados, podendo controlar infecções ocasionadas por Trypanosoma cruzi, Plamosdium chibaudi e Giardia duodenalis.

Palavras-chaves: Prevalência; Parasitoses; Lactobacillus.

\section{ABSTRACT:}

Intestinal parasitosis are diseases transmitted to humans, which contribute to various metabolic changes in the body of the host, these infections are associated with habits that enhance the process of dissemination of diseases and occur mainly in less developed regions, however it is suggested that probiotics, especially Lactobacillus spp. may increase the activity of intestinal, acting against parasitic infections. The present study is a bibliographic, qualitative review, where 39 references were used with the objective of evaluating the prevalence of enteroparasitosis and factors that determine this variation, as well as the effectiveness of Lactobacillus spp. in the control of parasitic infections. It was concluded that the prevalence of enteroparasitosis is related to socioeconomic and sanitary factors, and that although little is known about the mechanism of action, Lactobacillus spp. proved to be effective in the experiments performed, being able to control infections caused by Trypanosoma cruzi, Plamosdium chibaudi and Giardia duodenalis.

Keywords: Prevalence; Parasitoses; Lactobacillus. 


\section{INTRODUÇÃO}

Enteroparasitoses, também conhecidas como parasitoses intestinais, são doenças cujo agente etiológico apresenta ciclo evolutivo, localizam-se no aparelho digestório e são transmitidas aos seres humanos por meio da ingestão de cistos ou ovos presentes em alimentos contaminados (SILVEIRA, 2008). Dependem de outro organismo para se desenvolverem, sendo considerados assim como organismos oportunistas com potencialidade infecciosa que ocasiona danos à saúde do hospedeiro (GARZÓN et al 2015).

Nesse sentido, Brito (2003) também argumenta que as infecções parasitárias contribuem para diversas alterações metabólicas no corpo, sobretudo, a perda de micronutrientes, ocasionando principalmente desnutrição e quadro de anemia por deficiência de ferro.

Além disso, podem causar ainda quadros de diarreia, dores abdominais agudas, perda de peso, febre, náuseas e vômitos, entre outros sintomas, ou apresentar-se de forma assintomática. (ORLANDINI et al 2009; MOTTA et al 2002).

As crianças são as mais acometidas por doenças parasitárias, devido a práticas de higiene inadequadas e insuficiência do sistema imunológico (STRUFALDI et al 2003; MELO et al 2010). Infecções parasitárias infantis estão associadas a hábitos que potencializam o processo de disseminação da doença e podem desencadear um comprometimento do desenvolvimento físico e/ou intelectual e até mesmo complicações mais graves, tais como distúrbios respiratórios, hepatomegalia, ascite, coma e morte (ALVES et al 2005; PEDRAZA et al 2014).

Há uma variedade imensa de protozooses e helmintíases intestinais de relevância, que ocorrem principalmente em regiões menos desenvolvidas e sua incidência aumenta conforme piora as condições socioeconômicas (MELO et al 2010).

Sua prevalência está associada a instalações sanitárias inadequadas, poluição fecal da água e de alimentos a serem consumido, contato com animais e seus excrementos, saneamento básico, praticas educativas e de higienes, entre outros fatores (FLORES et al 2012; BELO et al 2012; BASUALDO et al 2007). 
Um estudo da Organização Mundial de Saúde publicados em 2005 calculou-se que mais de um bilhão de indivíduos no mundo, hospeda ao menos um tipo de espécie de parasitas intestinais, sendo Ascaris Lumbricoides a mais comum (WHO, 2005).

Devido a grande prevalência de enteroparasitoses em algumas regiões, o índice de óbitos por infecções parasitárias chegaram a ocupar o primeiro lugar, como no caso de países africanos e da América Latina (REY, 2008).

Contudo, a microbiota intestinal exerce grande influência sobre o bem-estar e ausência de doenças, atua sobre as diversas reações bioquímicas do hospedeiro e impede de maneira eficiente a ação de microrganismos potencialmente patogênicos, atuando no controle de infecções parasitárias (SAAD, 2006).

Alimentos suplementados com microrganismos vivos atuam na mucosa intestinal, aumentando a atividade da microbiota com propriedades úteis ao hospedeiro através da síntese de substâncias, esses alimentos são denominados probióticos e contém em sua composição bactérias, sendo as mais utilizadas o Lactobacillus spp. e Bifidobacterium spp. (HENKER et al 2007; VARAVALLO et al 2008).

Os probióticos geram efeitos positivos ao organismo, tais como, alívio dos sintomas causados por intolerância à lactose, tratamento de diarreia, aumento da resposta imune e efeitos anticarcinogênicos (FLESCH et al 2014)

O Lactobacillus spp. é uma bactéria bacilar, gram-positiva que atravessa a acidez do estômago e chega vivo ao intestino capaz de inibir a proliferação de microrganismos maléficos através da competição de locais de ligação, da produção de ácidos orgânicos e redução do pH intestinal (FLESCH et al 2014; BURITI et al 2007; GÓMEZ et al 2006).

Devido à sua elevada distribuição em território mundial, o presente trabalho procura avaliar a prevalência de enteroparasitoses e fatores que determinam a variação da sua frequência, além disso, busca verificar a eficácia do Lactobacillus spp. para o controle de infecções parasitárias, através de um estudo bibliográfico. 


\section{METODOLOGIA}

Trata-se de um estudo qualitativo de revisão bibliográfica, apropriada para discutir a prevalência das enteroparasitoses, e avaliar a eficácia do consumo do Lactobacillus spp. para o controle de parasitoses.

$\mathrm{Na}$ identificação das fontes bibliográficas foram utilizadas duas bases de dados: Literatura Latino-Americana e do Caribe em Ciências da Saúde (LILACS) e Scientific Electronic Library Online (Scielo). Foram consultados ainda livros e manuais publicados pelo Ministério de Saúde. Incluíram-se artigos nos idiomas português, inglês e espanhol cujos textos completos encontravam-se disponíveis para acesso publicados entre os anos 2003 e 2016, incluindo a literatura clássica.

As palavras-chave utilizadas foram "lactobacillus" "parasitoses" e "prevalência", suas correspondentes em inglês, "lactobacillus", "parasites" e "prevalence" e suas correspondentes em espanhol "lactobacillus, "parásitos" e "prevalencia"“.

Definidos os critérios de busca, realizou-se a coleta de dados, visando identificar os aspectos relevantes do tema disponíveis na literatura. Inicialmente, foi realizada a leitura dos títulos das publicações para verificar se os artigos atendiam aos critérios de inclusão. Na segunda fase, os resumos foram analisados para confirmar a utilização da publicação no estudo.

Foi utilizado um total de 39 referências, que foram lidas na íntegra e os aspectos relevantes foram fichados, permitindo observar a prevalência de pessoas afetas com parasitoses e o uso do lactobacillus spp. para o controle delas.

\section{RESULTADOS}

Dos artigos, 12 obtiveram dados importantes para responder o objetivo deste estudo, que foram organizados no quadro abaixo seguindo o critério de temática, seguindo a ordem de prevalência; edicácias de Lactobacillus spp. e benefícios dos probióticos. 
QUADRO 1 - Distribuição de referências, destacando autores, ano, objetivos e conclusões.

\begin{tabular}{|c|c|c|}
\hline AUTOR (ANO) & OBJETIVO & $\begin{array}{l}\text { CONCLUSÃO } \\
\end{array}$ \\
\hline $\begin{array}{l}\text { ALVES et al., } \\
2003\end{array}$ & $\begin{array}{l}\text { Avaliar a frequência de } \\
\text { enteroparasitoses em } \\
\text { localidades da área } \\
\text { urbana da cidade de São } \\
\text { Raimundo Nonato, Piauí. }\end{array}$ & $\begin{array}{l}\text { O parasito mais comum foi a Entamoeba coli }(35,8 \%) \text { seguido por } \\
\text { Endolimax nana }(13,6 \%) \text {, Hymenolepis nana }(9,4 \%) \text { e Ancilostomídeos } \\
(9,4 \%) \text {, foi observado ainda casos de infecções por Ascaris lumbricoides. }\end{array}$ \\
\hline $\begin{array}{l}\text { ORLANDINI et } \\
\text { al., } 2009\end{array}$ & $\begin{array}{l}\text { Observar a prevalência e } \\
\text { os fatores que favorecem } \\
\text { a proliferação de } \\
\text { parasitoses. }\end{array}$ & $\begin{array}{l}\text { A incidência de enteroparasitoses está diretamente relacionada à renda familiar, } \\
\text { observando maior prevalência em famílias com renda mensal de um salário } \\
\text { mínimo, além disso, observa-se que baixos índices de infecções estão associados } \\
\text { a fatores como consumo de água tratada e presença de rede de esgoto. }\end{array}$ \\
\hline $\begin{array}{c}\text { FLORES et } \\
\text { al., } 2012\end{array}$ & $\begin{array}{l}\text { Avaliar a prevalência de } \\
\text { enteroparasitoses em } \\
\text { crianças de grupos } \\
\text { escolares municipais da } \\
\text { cidade de Candida - BA. }\end{array}$ & $\begin{array}{l}\text { Das amostras obtidas, } 68,6 \% \text { foram negativas no exame parasitológico, } \\
\text { essa baixa prevalência pode estar relacionada com a adoção de } \\
\text { medidas profiláticas por parte dos participantes. Dos } 31,4 \% \text { de amostras } \\
\text { positivas, } 53,1 \% \text { continham Entamoeba coli. Entre os helmintos, o de } \\
\text { maior prevalência foi Enterobius vermiculares. }\end{array}$ \\
\hline $\begin{array}{l}\text { GARZÓN et } \\
\text { al., } 2015\end{array}$ & $\begin{array}{l}\text { Determinar a prevalência } \\
\text { de parasitas intestinais e } \\
\text { fatores de riscos em } \\
\text { crianças moradores de } \\
\text { assentamentos ilegais na } \\
\text { cidade de Florência- } \\
\text { Caquetá, Colômbia. }\end{array}$ & $\begin{array}{l}\text { As condições socioeconômicas interferem na prevalência de infecções } \\
\text { intestinais por parasitos, sendo de grande importância a intervenção } \\
\text { governamental para disponibilizar serviços de intervenção médica e } \\
\text { ações que melhorem a qualidade de vida da população. }\end{array}$ \\
\hline $\begin{array}{c}\text { MELO et al., } \\
2010\end{array}$ & $\begin{array}{l}\text { Verificar a importância da } \\
\text { realização de estudos } \\
\text { sobre a prevalência de } \\
\text { parasitos intestinais de } \\
\text { crianças em idade } \\
\quad \text { escolar. }\end{array}$ & $\begin{array}{l}\text { Considera parasitoses intestinais como um problema de saúde pública } \\
\text { que acarretam imensos prejuízos à saúde do hospedeiro, principalmente } \\
\text { em crianças devido a ineficácia do sistema imunológico, observando que } \\
\text { a prevalência de enteroparasitoses em crianças de idade escolar em } \\
\text { algumas regiões brasileiras pode variar de } 31 \text { a } 67 \% \text { dependendo do } \\
\text { nível socioeconômico e fatores como saneamento básico. }\end{array}$ \\
\hline $\begin{array}{c}\text { PINHEIRO, } \\
2011\end{array}$ & $\begin{array}{l}\text { Conhecer a relação entre } \\
\text { os determinantes sociais } \\
\text { e as parasitoses } \\
\text { intestinais e suas } \\
\text { consequências em } \\
\text { crianças. }\end{array}$ & $\begin{array}{l}\text { O grupo mais atingido por parasitos intestinais é o infanto-juvenil, devido a } \\
\text { diversos fatores como, por exemplo, a imunidade, nível de higiene, grau de } \\
\text { instruções dos pais, tipo de alimentação, tipo de instituição escolar que } \\
\text { frequenta, entre outros. As principais complicações foram anemia, } \\
\text { desnutrição, baixo rendimento escolar e baixo crescimento pondero-estatural, } \\
\text { sendo consequências de altas cargas parasitárias e constantes reinfecções. }\end{array}$ \\
\hline $\begin{array}{c}\text { BELO et al., } \\
2012\end{array}$ & $\begin{array}{l}\text { Analisar e descrever } \\
\text { fatores socioeconômicos, } \\
\text { demográficos e } \\
\text { ambientais associados à } \\
\text { ocorrência de } \\
\text { parasitoses intestinais. }\end{array}$ & $\begin{array}{l}\text { Notou-se que a presença de instalações sanitárias no domicilio foi } \\
\text { associadas a uma menor ocorrência de helmintos e que as } \\
\text { desigualdades nas condições de vida tornaram as prevalências } \\
\text { diferenciadas entre as regiões de estudo. }\end{array}$ \\
\hline $\begin{array}{l}\text { MAGALHÃES } \\
\text { et al., } 2013\end{array}$ & $\begin{array}{l}\text { Verificar a influência de } \\
\text { fatores socioeconômicos } \\
\text { na ocorrência de } \\
\text { enteroparasitoses e } \\
\text { orotozoários comensais em }\end{array}$ & $\begin{array}{l}\text { A prevalência de parasitos está associada a questões culturais, } \\
\text { socioeconômicas e sanitárias, tornando-se necessário o desenvolvimento de } \\
\text { campanhas preventivas como forma de romper o ciclo vicioso de tratamento } \\
\text { de reinfecções que sobrecarregam os gastos governamentais. }\end{array}$ \\
\hline
\end{tabular}

protozoários comensais em

áreas periféricas de Minas Gerais.

\begin{tabular}{|c|c|c|}
\hline $\begin{array}{l}\text { GÓMEZ et al., } \\
2006\end{array}$ & $\begin{array}{l}\text { Avaliar a capacidade do } \\
\text { Lactobacillus casei para } \\
\text { aumentar a resistência } \\
\text { contra infecções por } \\
\text { Plasmodium chabaudi. }\end{array}$ & $\begin{array}{l}\text { Lactobacillus casei aumenta a resistência inespecífica para Plasmodium } \\
\text { chibaudi, apresentando redução na parasitemia e viabilidade do parasito. }\end{array}$ \\
\hline $\begin{array}{l}\text { GARFIAS et } \\
\text { al., } 2008\end{array}$ & $\begin{array}{l}\text { Avaliar o efeito do } \\
\text { Lactobacillus casei na } \\
\text { infecção por } \\
\text { Trypanosoma cruzi. }\end{array}$ & $\begin{array}{l}\text { Tratamento com Lactobacillus casei via oral ou intraperitoneal induz uma } \\
\text { resposta protetora contra o Trypanosoma cruzi. }\end{array}$ \\
\hline $\begin{array}{l}\text { COÉLHO et } \\
\text { al., } 2016\end{array}$ & $\begin{array}{l}\text { Analisar a efetividade do } \\
\text { uso de Lactobacillus } \\
\text { casei Shirota para o } \\
\text { controle de giardíase em } \\
\text { crianças naturalmente } \\
\text { infectadas. }\end{array}$ & $\begin{array}{l}\text { Por meio do consumo da bebida láctea comercialmente disponível e } \\
\text { transcorrido } 21 \text { dias de tratamento, não houve detecção de formas } \\
\text { evolutivas de Giardia duodenalis nas fezes dos indivíduos, observando } \\
\text { assim uma melhora na consistência fecal, evidenciando o potencial de } \\
\text { L. casei para o controle de giardíase em crianças. }\end{array}$ \\
\hline $\begin{array}{l}\text { VARAVALLO } \\
\text { et al., } 2008\end{array}$ & $\begin{array}{l}\text { Salientar a importância } \\
\text { das bactérias probióticas } \\
\text { para a saúde humana. }\end{array}$ & $\begin{array}{l}\text { Bactérias probióticas são importantes para a saúde do homem e agem } \\
\text { através de muitos mecanismos de ação contra microrganismos } \\
\text { patogênicos. }\end{array}$ \\
\hline
\end{tabular}




\section{DISCUSSÃO}

A enteroparasitose é uma doença ocasionada por helmintos ou protozoários, que apresentam ciclos evolutivos e se instalam no intestino do indivíduo, provocando alterações patológicas (FERREIRA et al 2004). Flores et al (2012) destaca que esses parasitos apresentam-se em diversas formas, vários ambientes como habitat, são facilmente disseminados e capazes de infectar o homem, debilitando-o.

As parasitoses intestinais representam a doença mais comum no mundo, sendo corriqueiramente encontrado maior número de casos em países subdesenvolvidos (ORLANDINI et al 2009). Melo et al (2010) afirma que as parasitoses intestinais são consideradas um problema de saúde pública mundial, responsáveis por elevado índice de morbidade. Estudos realizados por Salomón et al (2007) revelam que regiões tropicais e subtropicais apresentam maiores taxas de parasitoses intestinais em relação a outras regiões.

Estima-se que as enteroparasitoses afetem cerca de 3,5 bilhões de pessoas, sendo maior dos casos em crianças (WHO, 2008). Por isso, torna-se necessário maior atenção, e medidas de controles específicos e direcionadas a população (BELO et al 2012; Fonseca, 2010; MELO et al 2010). Sabe-se ainda que a maior frequência na população infantil é devido a um sistema imunológico menos desenvolvido; hábitos de higiene de forma inadequada entre outros fatores (BELLOTO et al 2011; FLORES et al 2012).

A disseminação das diversas formas de enteroparasitos está relacionada diretamente a fatores como saneamento básico, nível socioeconômico, educação em saúde e outros aspectos pautados na qualidade de vida da população (MAGALHÃES et al 2013).

No Brasil, a distribuição de casos de infecções parasitárias varia de região para região, sendo a prevalência de infecções por protozoário maior que infecções por helmintos (BELO et al 2012). Análises realizadas por Flores et al (2012), apontam que a região Nordeste do país, onde as condições socioeconômicas são mais decadentes, dispõe de um maior número de casos, quando relacionado a outras regiões. Belo et al (2012) e Ferreira et al (2005) apontam que, áreas rurais brasileiras apresentam maiores números de casos de contaminações enteroparasitárias em relação às áreas urbanas.

Estudos ainda realizados por Flores et al (2012), na cidade de Candida, na Bahia, mostram que de 102 amostras submetidas a exame parasitológico de fezes, cerca de 31 , $4 \%$ foram diagnosticadas como amostras positivas, sendo que $56,25 \%$ correspondia ao sexo masculino e $43,75 \%$ ao sexo feminino. 
Magalhães et al (2013), em seu estudo realizado no interior de Minas Gerais, Brasil, também observou, assim como Flores et al (2012), que em relação ao sexo, o número de indivíduos homens parasitados é maior que o número de mulheres, e quanto maior o grau de escolaridade, menor o número de infecções.

Quanto a distribuição de espécie, observa-se através de estudos de Neves (2010) que o Hymenolepesis nana é mais frequente na região sul do país, neste sentindo, Flores et al (2012) destaca que Strongyloides stercoralis tem maior distribuição em áreas tropicais.

Segundo Giraldi et al (2001) os parasitas intestinais de maiores relevâncias e prevalência no Brasil são: Entamoeba histolytica, Balantidium coli, Schistosoma, Hymenolepis nana, Taenia, Ancylostoma duodenale, Ascaris lumbricoides, Enterobius vermiculares e Strongyloides stercoralis. E em relação à prevalência mundial de parasitismo, Ascaris lumbricoides é o helminto com maior frequência de infecção (VÉLEZ et al 2008).

Estudos realizados na cidade de Florência - Caquetá, na Colômbia, concluem que o protozoário mais comum é o Blastocystis spp. presente em $49,2 \%$ dos casos de infecções parasitárias, seguido por Giardia duodenalis (35,8\%) e Entamoeba histolytica (29\%), é possível observar ainda, que nas enteroparasitoses ocasionadas por helmintos, Ascaridíase é a mais frequente, seguida por Tricuríase (GARZÓN et al 2015).

Contudo estudos realizados por Henker et al (2007) apontam que os probióticos têm ações antiparasitárias, sendo denominados como alimentos funcionais, atuam na mucosa intestinal e a sua microbiota, alimentos como o iogurte, leites fermentados e alguns biscoitos estão inclusos neste grupo.

O Lactobacillus spp. é um dos principais probióticos, sendo comumente utilizados em indústrias de laticínios, aumenta as propriedades nutricionais dos alimentos (BURITI et al 2007; GÓMEZ et al 2006). Para Reid e Hammond (2005) eles agem através da competição por sítios de ligação, onde ocupa os receptores na mucosa intestinal, formando assim uma barreira física que impede que microrganismos patogênicos se liguem. Para Peluso (2007) os Lactobacillus spp. estimulam a resposta imune através do aumento da síntese de anticorpos, ativação de macrófagos, proliferação de células T e produção de interferon.

Matsumoto et al (2005) conclui em sua pesquisa, semelhantemente a Peluso (2007), que o consumo do Lactobacillus spp. fortalece o sistema imunológico através da produção de células de defesa. Teshima (2003) ainda enfatiza que eles aumentam a produção de enzimas que participam na digestão de lactose, sendo assim, seu consumo é 
importante para indivíduos que apresentam intolerância à lactose.

Também são responsáveis por liberarem substâncias, tais como, ácidos orgânicos, em especial o ácido lácteo, e peróxido de hidrogênio, que atuam contra infecções (VÉLEZ et al 2007). Outra funcionalidade dos lactobacillus spp. é a efetividade na atividade anticarcinogênico, ou seja, atuam na prevenção de neoplasias (NICOLAS et al 2007).

Em estudos realizados para a verificação da efetividade do Lactobacillus spp. Garfias et al (2008) conclui que a administração de Lactobacillus casei por via oral ou intraperitoneal mostra-se eficaz para o controle de infecção por Trypanosoma cruzi. Enquanto isso, Gómez et al (2006), em seu estudo realizado no México, sugere através de experimento prático que o Lactobacillus casei, quando administrado via intraperitoneal, aumenta a atividade fagocítica dos macrófagos, controlando não só o estagio inicial de infecções por Plasmodium chabaudi, como também a fase eritrocitária e quando alojados no baço.

Nesse mesmo objetivo, o estudo realizado com crianças brasileiras por Côelho et al (2016), revela a efetividade do Lactobacillus casei shirota para o controle de giardíase se ingerido diariamente por um período de vinte e um dias. $O$ autor ainda ressalta a importância da realização de novos estudos para comprovar a eficácia terapêutica, que uma vez constatada, torna-se um grande avanço para controle de cepas que apresentam resistências aos tratamentos alopáticos da indústria farmacêutica.

Nesse sentido, nota-se uma efetividade do consumo do Lactobacillus spp. contra infecções parasitárias, que são doenças comuns em território mundial, sobretudo em áreas subdesenvolvidas, sem condições sanitárias adequadas.

\section{CONSIDERAÇÕES FINAIS}

A partir deste estudo constatou-se que a prevalência de enteroparasitoses é relativa a diversos fatores, tais como, o clima e outras características da região; fatores socioeconômicos e sanitários e outras condições de vida, como por exemplo, práticas de higiene pessoal, e que, geralmente, o maior número de casos de infecções são em homens e crianças, além disso, pôde-se notar que áreas rurais têm um número maior de caso do que áreas urbanas.

Pôde observar também que embora, até esse tempo, haja poucos estudos, a utilização de um probióticos, em especial o Lactobacillus spp. tem eficácia frente à controle e prevenção de infecções parasitárias ocasionadas Trypanosoma cruzi, Plamodium 
chabaudi e Giardia duodenalis, contudo, necessita-se de estudos mais amplos para uma melhor avaliação de sua efetividade.

\section{REFERÊNCIAS}

ALVES, Rejane Maria de Souza; CARMO, Eduardo Hage. Plano Nacional de Vigilância e Controle das Enteroparasitoses. Secretaria de Vigilância em Saúde. Distrito Federal. 2005.

ALVES, Jair Rodrigues et al. Parasitoses intestinais em região semi-árida do Nordeste do Brasil: resultados preliminares distintos das prevalências esperadas. Cad. Saúde Pública, Rio de Janeiro. 19 (2): 667-670, 2003.

BASUALDO, Juan A. et al. Intestinal parasitoses and environmental factors in a rural population of Argentina, 2002-2003. Rev. Inst. Med. trop. S. Paulo, São Paulo, v. 49, n. 4, p. 251-255, Aug. 2007.

BELO, Vinícius Silva et al. Fatores associados à ocorrência de parasitoses intestinais em uma população de crianças e adolescentes. Rev. paul. pediatr., São Paulo , v. 30, n. 2, p. 195-201, jun. 2012.

BELLOTO, Marcus Vinicius Tereza et al. Enteroparasitoses numa população de escolares da rede pública de ensino do município de Mirassol - São Paulo, Brasil. Rev. Pan-Amaz Saude, vol. 2 (1), p. 37-44, 2011.

BRITO, L. L. et al. Fatores de risco para anemia por deficiência de ferro em crianças e adolescentes parasitados por helmintos intestinais. Revista Panamericana de Salud Pública, v. 14, n. 6, p. 422-431, dic. 2003.

BURITI, F.C.A; SAAD, Susana Marta Isay. Bactérias do grupo Lactobacillus casei: caracterização, viabilidade como probióticos em alimentos e sua importância para a saúde humana. Arch Latinoam Nutr 57: 373-380, 2007.

COÊLHO, Matheus Diniz Gonçalves et al. Avaliação do consumo de Lactobacillus casei Shirota para o controle de giardíase em crianças. Rev. Patol Trop. Vol 45 (2): 169-178. 2016. 
FERREIRA, Luiz Fernando; ARAÚJO, Adalto. Parasistismo, Doenças Parasitárias e Paleoparasitologia. In: COURA, José Rodrigues. Dinâmica das doenças infecciosas e parasitárias. Vol.1. Rio de Janeiro: Editora Guanabara Koogan S.A, 2005. Cap. 2, p. 7-18.

FERREIRA, J.R. et al. Diagnóstico e prevenção de parasitoses no reassentamento São Francisco, em Cascavel - Paraná. Revista Brasileira de Análises Clínicas, 36 (3), 145146, 2004.

FLORES, Manoel Carlos Prado et al. Prevalência de enteroparasitoses em crianças de grupos escolares municipais da cidade de Candida-Bahia. Bahia, 2012.

FLESCH, A. G; POZIOMYCK, A. K; DAMIN, D. C. O uso terapêutico dos simbióticos. Arq Bras Cir Dig. 2014.

FONSECA, E.O.L. et al. Prevalência e fatores associados ás geo-helmintoses em crianças residentes em municípios com baixo IDH no norte nordeste brasileiro. Caderno de Saúde Pública, v. 23, n.1, jan., 2010.

GARFIAS, Carlos Ramón Bautista; ÁLVAREZ, María del Carmen Torres; GÓMEZ, Martínez Federico. La inoculación de Lactobacillus casei em ratones NIH induce uma resposta protectora contra la infección por Trypanosoma Cruzi (cepa Ninoa). Vet. Méx., 39 (2), 2008.

GARZÓN, T. Lucero et al. Parasitosis intestinal y factores de riesgo en niños de los asentamientos subnormales, Florencia-Caquetá, Colombia. Revista Facultad Nacional de Salud Pública. 33 (2): 171-180, 2015.

GIRALDI, N. et al. Enteroparasites prevalence among daycare and elementar school children oh municipal schools, Rolânia, PR, Brazil. Revista da Sociedade Brasileira de Medicina Tropical, n. 34 (4), p. 385-387, 2001.

GÓMEZ, Federico Martínez; et al. Lactobaciilus casei ssp. rhamnosus enhances non specific protection against Plasmodium chabaudi AS in mice. Salud Publica Mex. 2006 Nov-Dec; 48(6):498-503. 
HENKER, J. et al. The probiotic Escherichia coli strain Nissle 1917 (EcN) stops acute diarrhoea in infants and toddlers. European Journal of Pediatrics, Berlin, v. 166, n. 4, p. 311-318, 2007.

MAGALHÃES, Thais da Rocha et al. Influência de fatores socioambientais na ocorrência de enteroparasitos e protozoários não patogênicos em área periférica do município de Cristina, MG - Brasil. Ver. Biociências, Taubaté, v. 19, n.2, p. 18-26, 2013.

MATSUMOTO, S. et al. Probiotic Lactobacillus-induced improvement in murine chronic 427 inflammatory bowel disease is associated with the down-regulation of pro-inflammatory cytokines 428 in lamina propria mononuclear cells. Clinical and Experimental Immunology, v. 140, n. 3, p. 429 417-426, 2005.

MELO Erenilson Moreira; FERRAZ, Fabiana Nabarro; ALEIXO, Denise Lessa. Importância do estudo da prevalência de parasitos intestinais de crianças em idade escolar. SaBiosRevista de Saúde e Biologia, [S.I.], v. 5, n. 1, ago. 2010.

MOTTA, Maria Eugênia Farias Almeida; SILVA, Gisélia Alves Pontes da. Diarréia por parasitas. Rev. Bras. Saude Mater. Infant., Recife , v. 2, n. 2, p. 117-127, ago. 2002.

NEVES, David Pereira, et al. Parasitologia Humana. 11ํㅡ. Ed. São Paulo: Editora Atheneu, 2010

NICOLAS, P. et al. M. V. Extensive horizontal transfer of core genome genes between two Lactobacillus species found in the gastrointestinal tract. BioMed Central Evolutionary Biology, London, v. 7, n. 141, p. 1-14, 2007.

PEDRAZA, D. F; QUEIROZ D; SALES, M. Doenças infecciosas em crianças pré-escolares brasileiras assistidas em creches. Ci Saúde Coletiva 19: 511-528, 2014.

PELUSO, I. et al. Lactobacillus paracasei subsp. paracasei B21060 suppresses human Tcell proliferation. Infection and immunity. Washington, v. 75, n. 4, p. 1730- 1737, 2007. 
PINHEIRO, Patricia Lopes. Enteroparasitoses na infância, seus determinantes sociais e principais consequências: uma revisão bibliográfica. Monografia de conclusão de curso - Universidade Federal de Minas Gerais, 2011.

ORLANDINI, Míriam Rossane; MATSUMOTO, Leopoldo Sussumu. Prevalência de parasitoses intestinais em escolares. Paraná, 2009.

REY, L. Parasitologia. Ed. Guanabara Koogan, 4ㅡㄹ. 2008.

REID, G.; HAMMOND, J. Probiotics: some evidence of their effectiveness. Canadian Family Physicians, Mississauga, v. 51, p. 1487-1493, 2005.

SAAD, S. Probióticos e prebióticos: o estado da arte. Revista Brasileira de Ciências Farmacêuticas, Tocantins, v. 42, n. 1, p. 1-16, jan. /mar. 2006.

SALOMÓN, M. et al. Prevalencia de Parásitos intestinales em niños de la ciudad de Mendoza, Argentina. Parasitol Latinoam. 2007. 62: 49-53.

SILVEIRA, Noelí Fátima. Estudo das enteroparasitoses correlacionando as condições sócio-econômicas e sanitárias de crianças que frequentam escolas de educação infantil públicas e privadas do município de Lajedo-Rs. 2008. 80 pgs. Monografia. UNIVASTES, Lajedo-RS.

STRUFALDI, Maria Wany Louzada et al. Prevalência de desnutrição em crianças residentes no Município de Embu, São Paulo, Brasil, 1996-1997. Cad. Saúde Pública, Rio de Janeiro, v. 19 , n. 2, p. $421-428$, Apr. 2003.

TESHIMA, E. Aspectos terapêuticos de probióticos, prebióticos e simbióticos. In: FERREIRA, C. L. L. F. Prebióticos e probióticos: atualização e prospecção. Viçosa: Viçosa, 2003.

World Health Organization. Report of the third global meeting of the partners for parasite control.: 2004 update. Geneva: WHO; 2005. 
World Health Organization. The global burden of disease.: 2004 update. Geneva: WHO; 2008.

VARAVALLO, M. A.; THOMÉ, J. N.; TESHIMA, E. Aplicação de bactérias probióticas para profilaxia e tratamento de doenças gastrointestinais. Semina: Ciências Biológicas e da Saúde, Londrina, v. 29, n. 1, p. 83-104, 2008.

VÉLEZ, H; ROJAS, W; BORRERO, J; RESTREPO, J. Enfermidades infecciosas. Fundamentos de Medicina. 6º ed. Corporación para Investigaciones Biológicas. 2008. 830.

*Autor para correspondência:

Franklin Emmanuel B. Pereira Filho

Email: franklinpereira57@gmail.com

Recebido: 17/10/2020 Aceite: 19/12/2020 